\title{
Plant diversity maintains long-term ecosystem productivity under frequent drought by increasing short-term variation
}

\author{
Cameron Wagg, ${ }^{1,2,8}$ Michael J. O’Brien, ${ }^{1,3}$ Anja Vogel, ${ }^{2,4,5}$ Michael Scherer-Lorenzen, ${ }^{6}$ \\ Nico Eisenhauer,${ }^{4,5}$ Bernhard Schmid, ${ }^{1}$ and Alexandra Weigelt ${ }^{4,7}$ \\ ${ }^{1}$ Department of Evolutionary Biology and Environmental Studies, University of Zürich, Winterthurerstr. 190, \\ Zürich CH-8057 Switzerland \\ ${ }^{2}$ Institute of Ecology, University of Jena, Dornburger Str. 159, Jena 07743 Germany \\ ${ }^{3}$ Estación Experimental de Zonas Áridas, Consejo Superior de Investigaciones Científicas, Carretera de Sacramento s/n, \\ E-04120 La Cañada, Almería, Spain \\ ${ }^{4}$ German Centre for Integrative Biodiversity Research (iDiv) Halle-Jena-Leipzig, Deutscher Platz 5e, Leipzig, Germany \\ ${ }^{5}$ Institute of Biology, Leipzig University, Deutscher Platz 5e, Leipzig 04103 Germany \\ ${ }^{6}$ Faculty of Biology, Geobotany, University of Freiburg, Schänzlestr. 1, Freiburg 79104 Germany \\ ${ }^{7}$ Institute of Biology, Leipzig University, Johannisallee 21, Leipzig 04103 Germany
}

Abstract. Increasing frequency of extreme climatic events can disrupt ecosystem processes and destabilize ecosystem functioning. Biodiversity may dampen these negative effects of environmental perturbations to provide greater ecosystem stability. We assessed the effects of plant diversity on the resistance, recovery and stability of experimental grassland ecosystems in response to recurring summer drought over $7 \mathrm{yr}$. Plant biomass production was reduced during the summer drought treatment compared with control plots. However, the negative effect of drought was relatively less pronounced at high than at low plant diversity, demonstrating that biodiversity increased ecosystem resistance to environmental perturbation. Furthermore, more diverse plant communities compensated for the reduced productivity during drought by increasing spring productivity compared to control plots. The drought-induced compensatory recovery led to increased short-term variations in productivity across growing seasons in more diverse communities that stabilized the longer-term productivity across years. Our findings show that short-term variation between seasons in the face of environmental perturbation can lead to longer-term stability of annual productivity in diverse ecosystems compared to less diverse ecosystems.

Key words: climate change; compensatory recovery; ecosystem resilience; insurance hypothesis; species loss; temporal scale.

\section{INTRODUCTION}

Extreme climatic events such as droughts affect terrestrial ecosystems across the globe and are predicted to increase in frequency under various global change scenarios (Dai 2013, Stocker et al. 2013). The severity of drought events and the associated water deficit depend on a combination of reduced precipitation intensity, duration and frequency as well as the seasonal timing of the events (Folke et al. 2004, Mitchell et al. 2016). Increased frequency of droughts during the season of high biological activity is anticipated to negatively impact ecosystem functioning in the long term, not only by directly disrupting ecosystem processes, but also by affecting the biodiversity that supports and maintains the functioning of the ecosystem in the first place (Folke et al. 2004, Cardinale et al. 2012, Hooper et al. 2012). Consequently, more frequent drought perturbations may be a considerable future climate-change threat for the long-term

Manuscript received 20 March 2017; revised 1 August 2017; accepted 24 August 2017. Corresponding Editor: Peter B. Adler.

${ }^{8}$ E-mail: cameron.wagg@ieu.uzh.ch functioning of ecosystems and the services they provide for society (Schröter et al. 2005, Lehner et al. 2006).

The ability of an ecosystem to maintain stable functioning in the face of frequently recurring summer drought depends on its resistance during and its recovery after drought perturbation. An ecosystem can achieve high resistance by minimizing losses in functioning during drought and high recovery by restoring functioning after drought; the two key features of ecosystem resilience (Schläpfer and Schmid 1999, Oliver et al. 2015). The resistance and recovery of ecosystems may be influenced by community characteristics such as species diversity, composition and functional traits (van Ruijven and Berendse 2010, Isbell et al. 2015, Skelton et al. 2015, Fischer et al. 2016). For instance, greater diversity is likely to stabilize ecosystem functioning against an extreme event because more diverse communities are more likely to include some species that will be able to maintain ecosystem functioning, known as the insurance hypothesis (Doak et al. 1998, Schläpfer and Schmid 1999, Yachi and Loreau 1999). The importance of such population and species level compensatory effects for mediating ecosystem stability has been shown both empirically and 
theoretically (Gonzalez and Loreau 2009, Loreau 2010, de Mazancourt and Loreau 2013, Gross et al. 2014, O'Brein et al. 2017). Diversity has also been found to have significant to non-significant effects on the resistance against the loss of productivity during drought, or the productivity recovered after drought (Tilman 1996, Pfisterer and Schmid 2002, Wang et al. 2007, van Ruijven and Berendse 2010, Vogel et al. 2012, Isbell et al. 2015). Consequently, the role that biodiversity plays in stabilizing the long-term productivity of an ecosystem under recurrent summer droughts remains largely untested with most studies assessing the effects of a single drought event and short-term resistance and recovery (Cardinale et al. 2012, Hooper et al. 2012, Oliver et al. 2015, 2016). However, understanding how short-term resistance and recovery responses maintain the long-term productivity of an ecosystem still requires long-term empirical observations (Oliver et al. 2015).

The stability of productivity can be defined as the inverse coefficient of variation (the mean relative to the standard deviation). Thus stability is codetermined by both the temporal variation in productivity as well as the overall mean in productivity (Lehman and Tilman 2000, Gross et al. 2014, and see Fig. 1). Resistance and recovery responses to recurring perturbations can determine the stability through altering the mean or the variation in productivity over time (Gross et al. 2014, Isbell et al. 2015, Oliver et al. 2015). For instance, an ecosystem that has high resistance will be only marginally destabilized due to the minimal influence of the drought on its productivity (Fig. 1a). An ecosystem that has a lower resistance and fully recovers before the next event will be destabilized due to a reduced mean productivity and an increased temporal variation in productivity (Fig. 1b). When an ecosystem has both low resistance and low recovery, recurrent perturbations can destabilize the system by reducing the mean productivity without greatly altering its temporal variation (Fig. 1c). Finally, an ecosystem may maintain its mean productivity by increasing productivity during recovery following the drought to compensate for lost productivity during drought events, which we refer to as "compensatory recovery." This would destabilize short-term productivity due to the greater temporal variation (Fig. 1d). In this last scenario, when the productivity oscillates around the mean of non-perturbed conditions, the ecosystem is considered to have a low resilience since the productivity of the system is greater in comparison with non-perturbed conditions after the perturbation event and has thus not returned to its non-perturbed state (Isbell et al. 2015). However, over longer temporal scales, the ecosystem is stabilized through the short-term responses that maintain the mean productivity, such as through greater resistance (e.g., Fig. 1a) to perturbation or compensatory recovery (e.g., Fig. 1d).

To test whether greater plant diversity maintains a more stable long-term productivity and whether this is due to the short-term resistance and recovery responses to drought perturbations, we assessed the response of primary productivity during and after recurrent summerdrought events over $7 \mathrm{yr}$ in experimental grassland communities. Using paired drought and non-drought (ambient) plots that had the same initial plant community composition, we assessed how resistance and recovery in response to drought events contributed to stabilization, or variation in ecosystem functioning, within and across years, as a function of plant diversity. We found that more species-rich ecosystems were more resistant to summer drought (e.g., Fig. 1a) and also showed compensatory recovery such that the long-term mean in productivity was maintained. That is, high biodiversity buffered against losses in productivity under drought events by increasing productivity the following spring. The maintenance of the long-term mean in productivity was further enhanced through greater resistance to drought events of more diverse compared with less diverse ecosystems. These results demonstrate that diversity can maintain stable long-term annual productivity through compensatory recovery that increases short-term variations in productivity in response to perturbation.

\section{Materials And Methods}

\section{Experimental design}

The experiment was set up within the Jena Experiment (details and data available at http://www.the-Jena-Expe riment.de). The plant communities natural to the area are primarily dominated by perennial plant species, particularly grasses, and peak biomass typically occurs in late spring (Roscher et al. 2004, also see Fig. 2). The mean annual precipitation for the site during our experimental period was $595.7 \mathrm{~mm}(\mathrm{SD}=61.5 \mathrm{~mm})$ and the growing period typically began in late March (daily average temperature $10^{\circ} \mathrm{C}$ or greater for three consecutive days, see Table S1 and Fig. S1). Plant communities used in the experiment were initially sown in 2002 using a pool of 60 common, largely perennial grassland species and were sown in equal proportions into 80 plots with species richness levels of $1,2,4,8,16$, and 60 species (Roscher et al. 2004). Plots occur across four blocks within the field based on edaphic characteristics (Roscher et al. 2004). Communities were maintained by weeding every spring, summer, and autumn and by mowing in spring and late summer directly following the biomass harvest (see Weisser et al. 2017).

Our study was initiated in 2008 and utilized 76 plots with two $1 \times 1 \mathrm{~m}$ subplots designated as either drought or ambient control. Rainout shelters were set up midsummer over plant communities sown with 1, 2, 4, 8, or 16 plant species. Each diversity level was replicated with 16 different species compositions (plots) with the exception of monocultures and 16-species mixtures with 14 compositions. Rainout shelters were constructed using wooden frames with transparent PVC roofs (see Vogel et al. 2013 for further construction details). In 2008, 

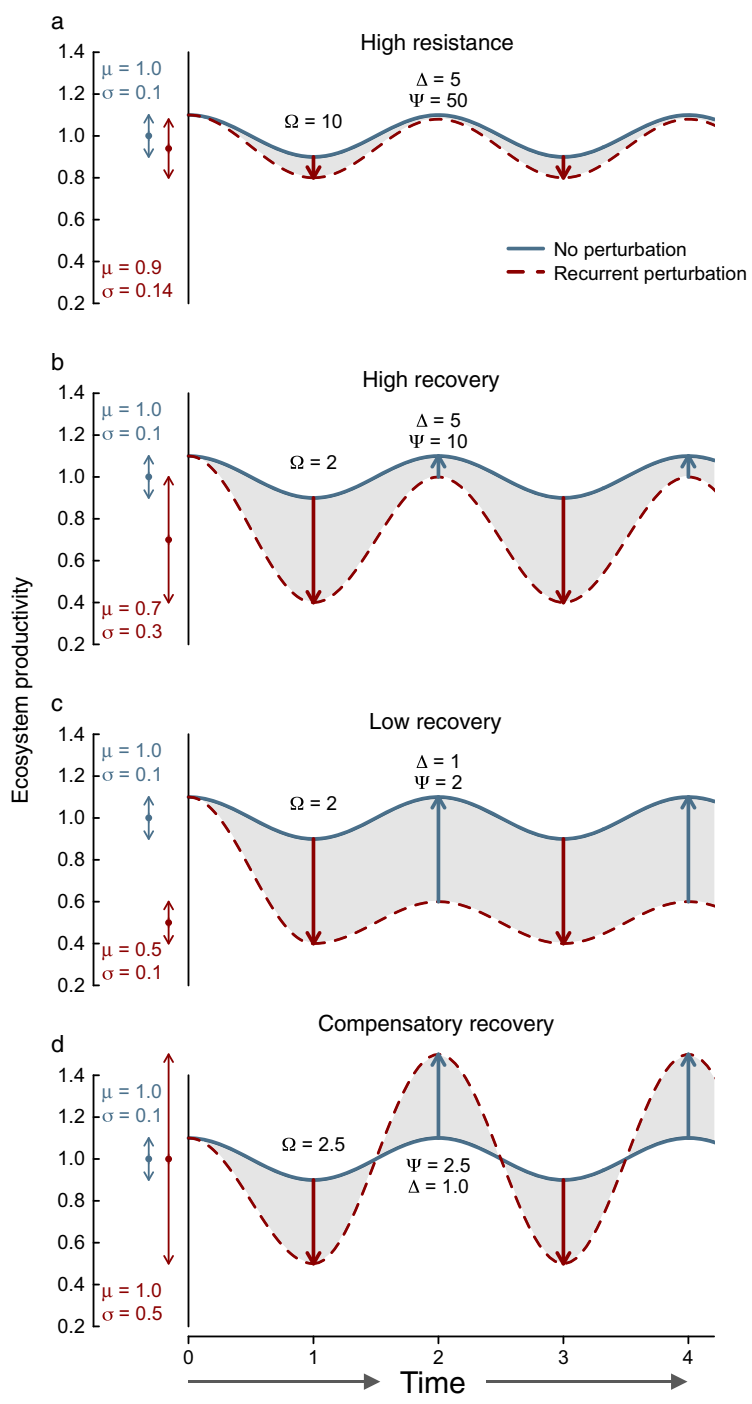

shelters were only constructed over drought subplots, but from 2009 onward shelters were constructed over both subplots to account for any potential shading effect the roof may have imposed (Vogel et al. 2013). Rainwater was collected in rain barrels and used to water ambient subplots following rainfall events with the same rainfall per $\mathrm{m}^{2}$ (see Vogel et al. 2012, 2013). Shelters excluded natural rainfall from mid-July to the end of August (six weeks). The rain withheld from the drought subplot reduced the frequency and amount of summer precipitation by $43 \%$ and $42 \%$, respectively (see Table S1, Fig. S1 and Fig. 2). In the final year of our study (2014), 37 of the 78 plots (47\%) had lower realized species richness in the drought than in the paired ambient subplot. However, this did not drastically alter species richness and the sharp richness gradient was maintained.

Standing biomass was harvested in May, 6 weeks before the installation of shelters, and again 6 weeks after the installation of shelters, when they were removed
FIG. 1. Examples by which a system may respond to drought perturbations that can destabilize the functioning of the system (e.g., ecosystem productivity) over time depending on the resistance to perturbation $(\Omega$, indicated by the downward-pointing red arrows) and the recovery from perturbation ( $\Psi$, indicated by the upward-pointing blue arrows). Both resistance and recovery determine the systems resilience $(\Delta=\Psi / \Omega$, see Methods). The solid blue line indicates the functioning of a non-perturbed system (ambient conditions) with an overall unit mean $(\mu=1)$ and varying by $10 \%$ around the mean $(\sigma=0.1)$ in all cases. The dashed red line indicates the functioning of a system experiencing recurrent perturbation (i.e., between time points 0 and 1 , or, 2 and 3 ) followed by a period of recovery (i.e., between time points 1 and 2, or, 3 and 4). The grey-shaded region highlights the difference between the two. The overall mean $(\mu)$ and variation $(\sigma)$ in the functioning of a system through time is indicated on the left by the point and doublehead arrow, respectively (and values are provided). In (a) the system exhibits high resistance to perturbation where productivity is not strongly affected by drought perturbation ( $\mu$ and $\sigma$ are only slightly altered). In (b) the system exhibits a high recovery resulting from the ability to rapidly return to unperturbed conditions following perturbation, such that $\Psi>\Omega$, which results in an overall lower mean $(\mu)$ and greater variation $(\sigma)$. In $(c)$ the system exhibits low resilience following perturbation such that $\Psi \approx \Omega$, where $\mu$ is reduced, but $\sigma$ is unchanged. In (d) the system is destabilized by the increase in variation only that results from compensatory recovery ( $\mu$ remains unchanged, but $\sigma$ is increased), resulting in a lower resilience of the system because the functioning of the system still deviates above the non-perturbed conditions where $\Psi \approx \Omega$. Data are simulated by the function $y=\mu+\sigma^{*} \cos \left(\right.$ Time $\left.^{*} \pi\right)$.

(August). Plant communities were left under natural conditions from September until the following summer. Sown plant species were separated from weed species, and the dead plant material was also separated out. The sown plant species biomass was dried at $70^{\circ} \mathrm{C}$ for $48 \mathrm{~h}$ and weighed to quantify ecosystem productivity, representing regrowth since the previous cutting.

The relative response in productivity of drought subplots relative to ambient subplots was calculated for each season using the $\log$-response ratio, $\log \mathrm{R} R=\log (x)$ $\mu)$. Here, $x$ is the productivity in a drought subplot and $\mu$ is the temporal mean productivity in the paired ambient subplot for a given season (i.e., $\mu_{\text {Summer }}$ or $\mu_{\text {Spring }}$ ). The temporal mean of the paired ambient subplot was used instead of the productivity of a single time point to avoid 0 or missing values in the denominator. Additionally this relative response calculation parallels the calculations of stability, resistance, and recovery (see below). The long-term relative response was calculated in the same manner where $x$ is the annual net productivity in a drought subplot and $\mu$ is the mean in the annual net productivity in the paired ambient subplot over all years.

\section{Stability, resistance, recovery and resilience}

The variation in seasonal productivity was calculated as the inverse coefficient of variation $(\mu / \sigma)$, commonly referred to as temporal stability (Lehman and Tilman 2000). Here, $\mu$ is the temporal mean in productivity of a subplot across seasons and $\sigma$ is seasonal variation in 


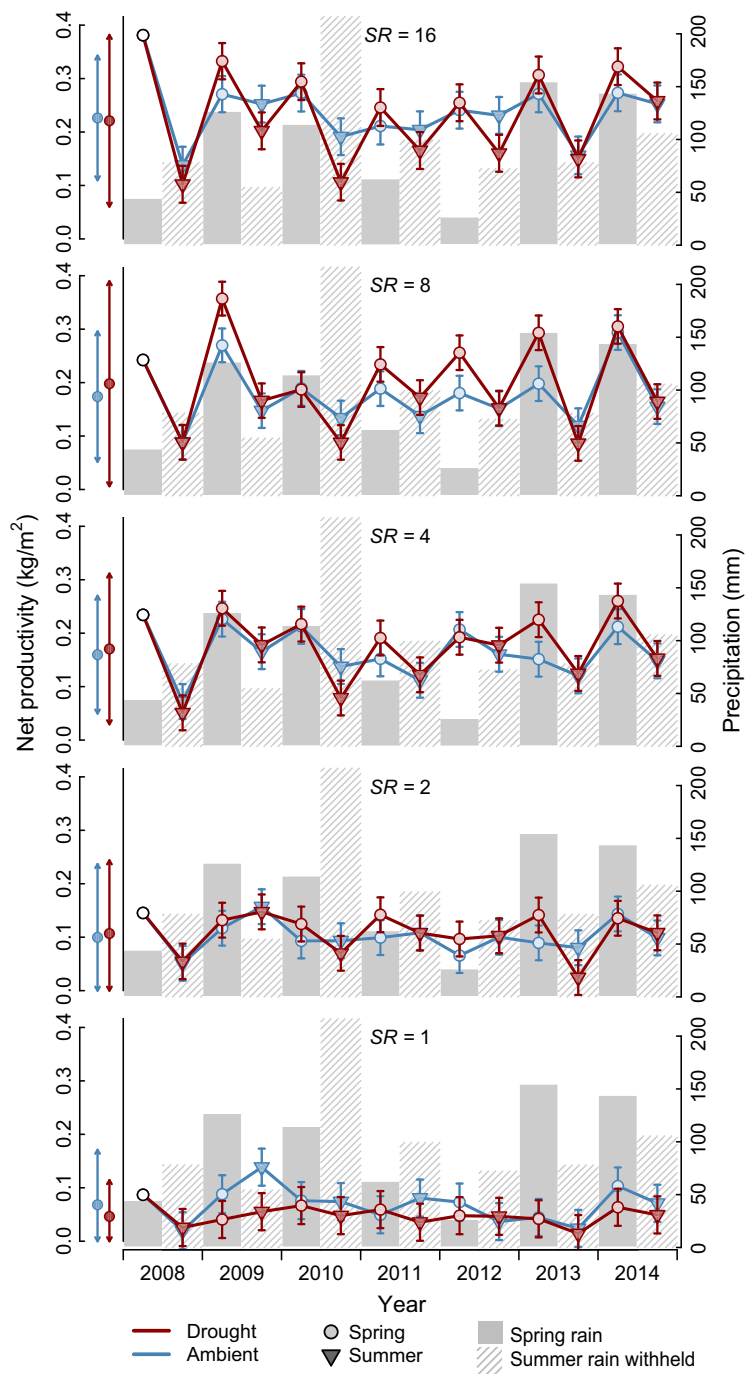

FIG. 2. Mean productivity for each species richness level (SR) under drought and ambient conditions during spring and summer of each year are shown. Red and blue lines interconnecting means highlight the temporal trend. Error bars indicate the standard error of the model mean estimates. Points and arrows on the left are the overall temporal mean and standard deviation. Grey shaded bars in the background indicate the amount of rain received in the spring (between the first growing degree day of the year and the installation of rainout shelters; solid bars) and the amount of rain withheld from the drought plots by the rainout shelters over the 6-week summer period (hashed bars). The drought was initiated in the summer of 2008 , and the open point indicates the average spring 2008 productivity prior to the initial drought.

productivity of a subplot. The stability in annual productivity was calculated in the same manner, but using the mean and variation in annual productivity (sum of spring and summer productivity). Since stability is related to the deviation in productivity from ambient conditions during drought and following a period of recovery we calculated the resistance $(\Omega)$, recovery $(\Psi)$ and resilience $(\Delta)$ following Isbell et al. (2015) to understand the effect of diversity on the seasonal variation under recurrent summer droughts. Resistance is calculated as $\Omega=\mu_{\text {Summer }} /\left|x_{\text {Summer }}-\mu_{\text {Summer }}\right|$, where $x_{\text {Summer }}$ in the calculation is the productivity under drought conditions directly following the imposed summer drought perturbation and $\mu_{\text {Summer }}$ is the temporal mean productivity of the paired ambient subplot. Similarly, recovery is calculated as $\Psi=\mu_{\text {Spring }} / \mid x_{\text {Spring }}-\mu$ Spring, where $x$ Spring is the spring productivity in a drought subplot and $\mu_{\text {Spring }}$ is the mean productivity in the ambient conditions. Finally, resilience is calculated as the proportion of recovery to resistance $(\Delta=\Psi / \Omega)$, paralleling the definition of resilience outlined elsewhere, where the deviations in productivity following a period of recovery from perturbation is proportional to the deviations in productivity resulting from perturbation (Lloret et al. 2011, Isbell et al. 2015).

In our study, the indices of resistance $(\Omega)$ and recovery $(\Psi)$ reflect the relative deviations in productivity under drought conditions from ambient conditions, such that larger values indicate smaller relative differences in productivity between communities under drought and ambient conditions (see Fig. 1). Importantly, these indices of resistance and recovery differ from the relative response since they do not distinguish between differences above or below productivity under ambient conditions. The two measures parallel the index of stability by dividing the temporal mean $(\mu)$ by the average absolute difference from the mean (i.e., similar to $\sigma$ ) allowing for resistance, recovery and stability to be readily compared among each other and with other studies on a common relative scale. Although previous definitions and calculations of ecosystem resilience vary and have generated some debate (Schläpfer and Schmid 1999, Hodgson et al. 2015, Mori 2016, Oliver et al. 2016), here it quantifies the proportion of drought-induced reduction of summer productivity that was recovered again the following spring. For instance, in Fig. $1 b, \Omega=2$ and $\Psi=10$ (and thus $\Delta=5$ ) indicates that the community has recovered to $80 \%$ of its functioning that was lost during the drought compared to the ambient non-perturbed community (calculated as $\left.1-\Delta^{-1}\right)$. Furthermore, if $\Delta=1$, then the community under drought conditions deviates equally from the ambient conditions in both spring and summer, such that the ecosystem has not returned to its non-perturbed state following a recovery period (i.e., Fig. 1c, d).

\section{Analyses}

All statistical analyses were done with $\mathrm{R}$ version 3.1.2. Mixed-effects models were fitted with the $\mathrm{R}$ package "ASReml" (VSN International Ltd.). For all analyses, we only considered data from 2009 onwards in order to have paired spring and summer responses within each year, as well as to avoid potential effects resulting from the different setup in 2008 (see Methods and Fig. 2 for 2008 data; Vogel et al. 2013). In all models, block was included as a fixed-effects factor (Schmid et al. 2017). 
Further fixed-effects terms were diversity (log-richness), drought treatment (drought vs. ambient control), season (spring vs. summer), year (6-level factor) and all interactions among these. Random-effects terms included plot, subplot within plot, plot $\times$ year, plot $\times$ season, and plot $x$ year $\times$ season. Residuals were corrected for serial correlations over time by fitting an auto-regressive structure along all 12 ordered season $\times$ year time points at the subplot level. Seasonal productivity was analyzed as first dependent variable. The seasonal relative response between drought and control plots was also analyzed with the above model, but without terms that included drought and without the subplot as random-effects term. Annual productivity was analyzed with a model excluding season and its interactions as explanatory terms. Finally, the annual relative response was analyzed in the same way as the seasonal relative response, but again without season and its interactions.

The stability of seasonal and annual productivities was analyzed with the model introduced above, but without terms involving season or year. To further understand the effects of diversity on stability under recurrent droughts, we "unpacked" the diversity-stability relationships into their component parts by assessing the log-log relationships of $\mu, \sigma$ and $\mu / \sigma$ with species richness. The coefficients $\beta \mu$ and $\beta \sigma$ from these regressions indicate the effect size of species richness on $\mu$ and $\sigma$ that determine the effect of species richness on stability $\left(\beta_{C V}\right)$, because $\beta_{C V}$ is conveniently the difference of $\beta \mu$ and $\beta \sigma$ (see Gross et al. 2014). Resistance, recovery and resilience were log-transformed prior to analysis and assessed for their relationship with diversity by regression, using plot and year as random terms.

\section{RESULTS}

\section{Short- and long-term responses to drought}

The recurrent summer droughts had a strong effect on productivity depending on the season (drought $\times$ season interaction: $F_{1,74.0}=33.32, P<0.001$, Table S2). During the summer, when the drought treatment was imposed, productivity was reduced under drought compared to ambient conditions (drought $=0.118 \mathrm{~kg} / \mathrm{m}^{2}$, ambient $=$ $0.134 \mathrm{~kg} / \mathrm{m}^{2}, \mathrm{SE}=0.0079, P=0.001$, Fig. 2, Table S2). Conversely, the spring productivity under drought was overall greater than under ambient conditions (drought $=$ $0.195 \mathrm{~kg} / \mathrm{m}^{2}$, ambient $=0.169 \mathrm{~kg} / \mathrm{m}^{2}, \mathrm{SE}=0.0079, P<$ 0.001 , Fig. 2, Table S2). As a consequence of the increased spring productivity, there was no overall effect of drought on the long-term average in productivity across all seasons (net drought effect: $F_{1,73.8}=0.76, P=0.387$, Fig. 2, Table S2). This also resulted in recurrent summer droughts having no overall effect on the long-term average in annual productivity $\left(F_{1,72.7}=0.58, P=0.449\right.$, Table S3).

Summer droughts also did not significantly alter the diversity-productivity relationship during the summer
(Fig. 3a, drought vs. ambient slope: $P=0.861$ ) and the relative response to drought in summer only increased non-significantly with plant diversity (Fig. 3b). However, the recovery after summer drought increased with increasing diversity, as indicated by the significantly greater spring productivity compared to the ambient treatment in both absolute (Fig. 3c) and relative terms (Fig. 3d). Thus, recurrent droughts altered the diversityproductivity relationship in the spring, but not during summer drought (season $\times$ diversity $\times$ drought interaction: $F_{1,74.2}=6.31, P=0.014$, Table S2). The relationship between diversity and the long-term annual productivity was marginally steeper under recurrent drought compared to communities under ambient conditions (Fig. 3e, diversity $\times$ drought interaction: $F_{1,72.9}=3.64, P=0.060$, Table $\mathrm{S} 3$ ). This became significant if analyzed in relative terms, where the response to drought in the long-term annual productivity changed from negative at low to slightly positive at high diversity (Fig. 3f, $F_{1,71}=7.56, P=0.007$ ).

\section{Short-term stability}

As a consequence of the elevated spring productivity in more diverse plant communities under drought conditions, the droughts resulted in a weaker diversity-stability relationship compared with ambient conditions at the short-term seasonal scale (Fig. $4 \mathrm{a}, F_{1}, 74=12.12$, $P<0.001)$. However, even in communities experiencing summer droughts, greater diversity still had a significantly more stabilizing effect on productivity than did lower diversity (Fig. 4a).

The "unpacking" of the diversity-stability relationship into the effects of diversity on the short-term seasonal $\mu$ and $\sigma$ of productivity ( $\beta \mu$ and $\beta \sigma$, respectively) revealed that diversity increased stability by increasing mean productivity more than it increased its variation $(\beta \mu>\beta \sigma)$ in both the communities experiencing recurrent droughts $(\beta \mu=0.694, \mathrm{SE}=0.079, \quad P<0.001$ and $\beta \sigma=0.425$, $\mathrm{SE}=0.072, P<0.001)$ and in communities under ambient conditions $(\beta \mu=0.521, \mathrm{SE}=0.079, P<0.001$ and $\beta \sigma=0.181, \mathrm{SE}=0.072, P=0.012)$. Nevertheless, the difference in the effect of diversity on the variation of productivity between drought and ambient conditions (drought $\beta \sigma$ - ambient $\beta \sigma=0.244$ ) was greater than the difference in the effect of diversity on mean productivity between the two treatments (drought $\beta \mu$ - ambient $\beta \mu=0.173$ ), indicating that at higher diversity drought had a stronger effect on the short-term seasonal variation of productivity than on its mean (Fig. 2).

\section{Long-term stability}

The positive effect of diversity on the long-term stability of the annual net productivity was again due to stronger positive effects on increasing the mean than on reducing the variation in the annual net productivity under both drought and ambient conditions. Although 


\section{Summer}
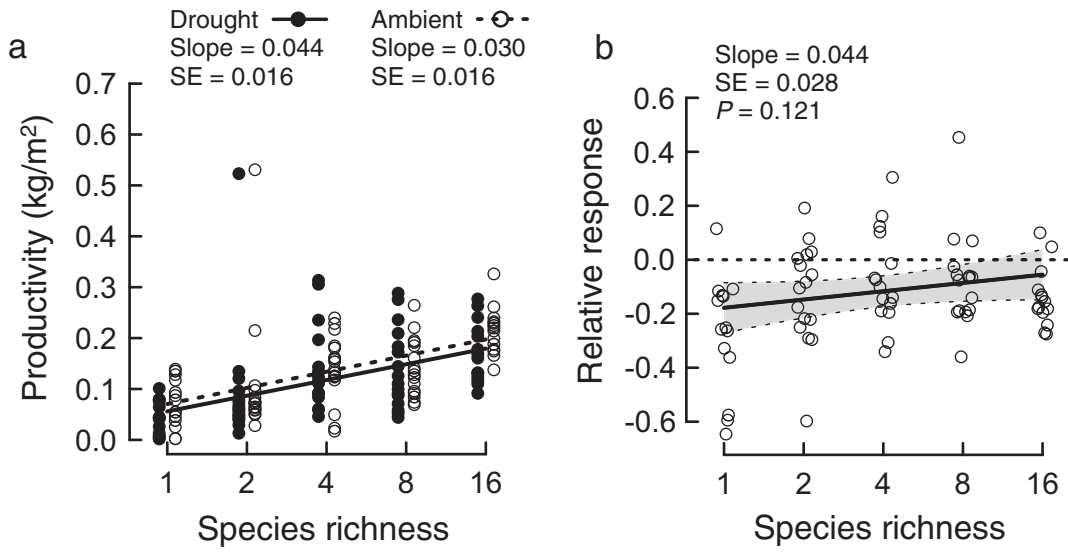

Spring
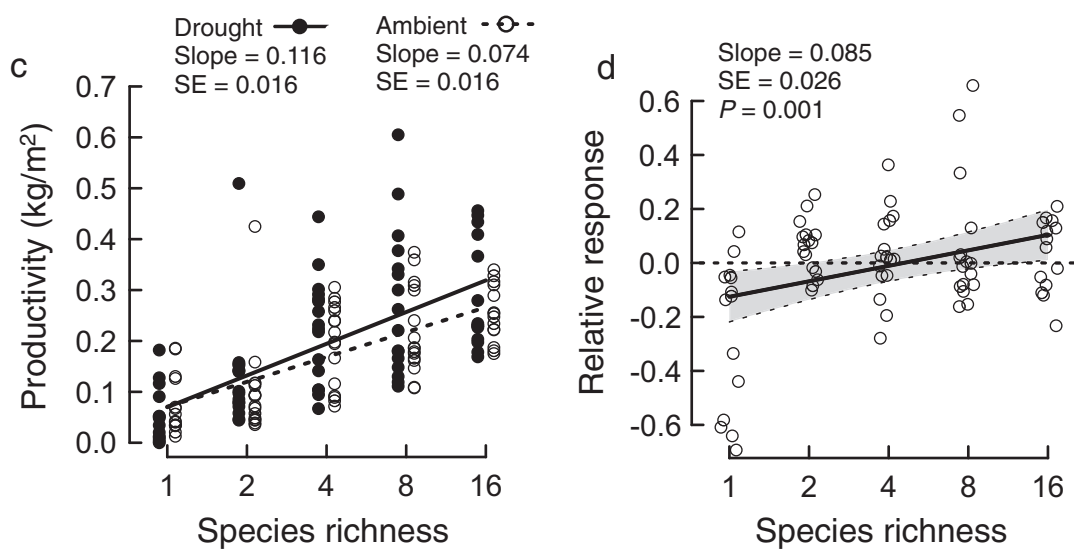

\section{Long-term}
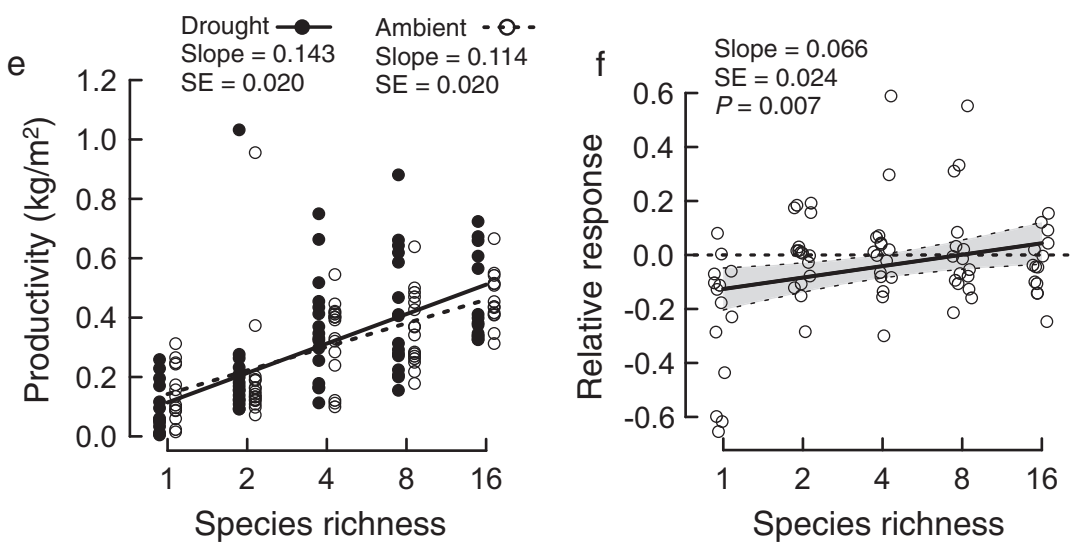

FIG. 3. The effect of drought on the communities' short-term productivity shown for (a) the temporal mean productivity in both drought and ambient treatments and (b) the relative response in relation to increasing plant species richness. The effect of drought on the communities in the spring following a period of recovery is shown for (c) the temporal mean productivity in both drought and ambient treatments and (d) the relative response in relation to increasing plant species richness. The annual productivity (sum of spring and summer) under drought and ambient conditions in relation to richness is shown in absolute (e) and in relative terms (f). Points indicate individual communities and the relative response on the right is the log-ratio of productivity under drought relative to ambient. Slopes and standard errors (SE) for all relationships are provided. All diversity-productivity slopes were highly significant $(P<0.001)$. $P$-values are provided for the significance of the diversity-relative response slopes and the $95 \%$ confidence interval around the diversity-relative response relationships is shaded in grey. Species richness axes are log-scale. 

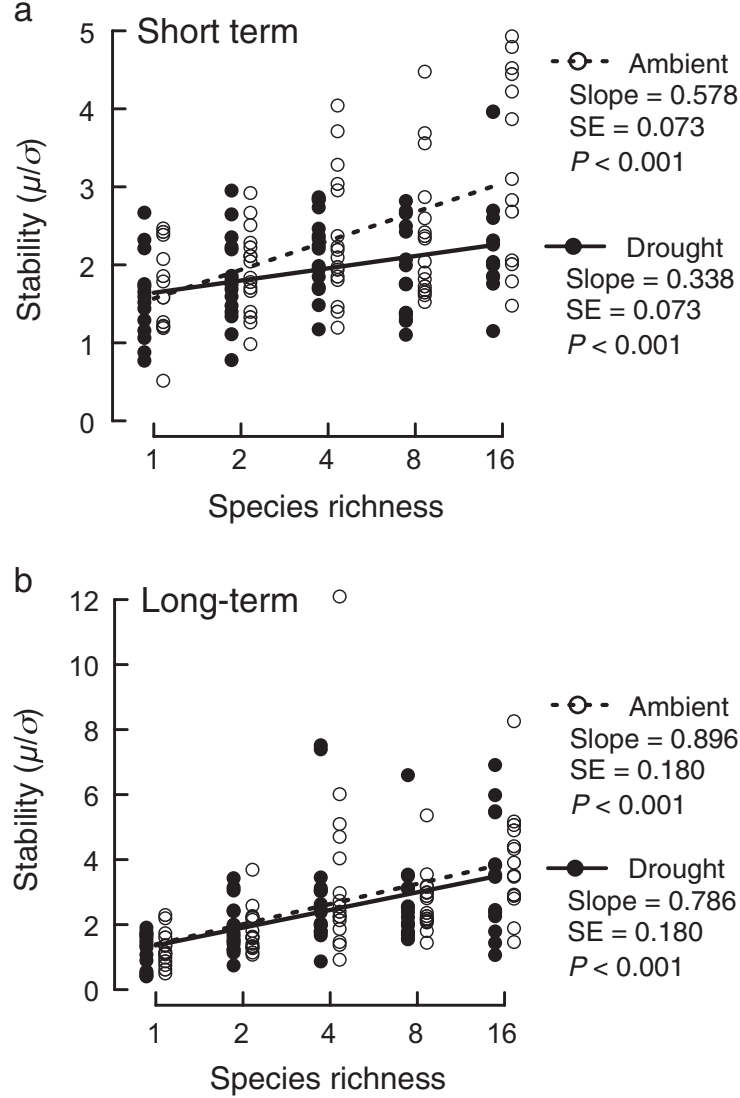

FIG. 4. The effects of plant species richness on the stability of community productivity on the short-term scale across seasons (a) and the long-term scale across years (b) are shown, where larger values indicate smaller variation. Slopes, standard errors (SE) and $P$-values of all relationships are provided. Species richness axes are log-scale.

the effect of diversity on the variation of the annual net productivity was larger under recurrent droughts (drought $\beta \sigma=0.338, \mathrm{SE}=0.080, P<0.001$ ) than under ambient conditions (ambient $\beta \sigma=0.138, \mathrm{SE}=0.080$, $\left.P=0.052 ; F_{1}, 74=6.79, P=0.011\right)$, it was not great enough to significantly alter the diversity-stability relationships between drought and ambient conditions (Fig. $4 \mathrm{~b}, F_{1,74}=0.26, P=0.609$ ).

\section{Resistance, recovery and resilience}

In communities that experienced recurrent droughts, the resistance to summer drought increased significantly with plant species richness from $1.63(\sim 61 \%$ difference in biomass) in monocultures to 2.79 ( $36 \%$ difference) in 16 -species mixtures (Fig. 5a, $F_{1,71.6}=10.15, P=0.002$ ). Similarly, the spring recovery also increased with species richness from 1.89 to 4.10 (Fig. $5 \mathrm{~b}, F_{1}, 71.5=11.82$, $P<0.001)$. However, resilience did not change with plant species richness and never significantly differed from a value of 1 (Fig. 5c, $F_{1,74}=1.10, P=0.298$, slope $=0.095)$.

\section{Discussion}

Our study demonstrates that diversity can maintain long-term annual productivity in the face of recurrent summer droughts over $7 \mathrm{yr}$ by increasing the short-term variation in seasonal productivity. Specifically, we found that summer droughts had little effect on the diversityproductivity relationship during summer droughts, but instead strengthened the diversity-productivity relationship in the spring. This was because all plant communities suffered similar reductions in absolute productivity during drought, regardless of the number of species, but in the following spring, more diverse communities were better able to compensate for losses in summer productivity (compensatory recovery, i.e., Fig. 1d). This compensatory recovery phenomenon was previously observed in the first year in a similar experiment using the same plant communities where diversity increased the productivity following a period of recovery after drought, but only under a more intense management regime with high $\mathrm{N}$ fertilization (Vogel et al. 2012). Here we show that this compensatory recovery pattern is not a single-event phenomenon dependent on management, but is rather the short-term mechanism that provides stability to the long-term productivity in more diverse communities.

Although the drought led to diversity having a weaker effect on the stability across seasons (compared to ambient conditions), we found higher plant diversity had a positive effect on stability under both drought and nondrought conditions as well as at both the short-term and long-term timescales. These general results provide further support to the growing number of studies that greater diversity supports greater stability in ecosystem functioning (Tilman and Downing 1994, Schläpfer and Schmid 1999, Lehman and Tilman 2000, Pfisterer et al. 2004, van Ruijven and Berendse 2010, Isbell et al. 2015, Oliver et al. 2015).

Although productivity was similarly reduced by drought in all communities in absolute terms, the more productive and diverse communities suffered smaller relative losses. Consequently, the stabilizing effect of diversity in communities experiencing recurrent droughts resulted from greater resistance to drought in support of previous findings (e.g., Tilman and Downing 1994, Isbell et al. 2015). The positive diversity-resistance and diversity-recovery relationships show that more diverse communities deviated relatively less in productivity from ambient conditions during summer droughts (resistance) and in the following spring (recovery) compared to less diverse communities. Thus, under recurrent drought conditions, greater diversity still provided a more stable long-term productivity relative to less diverse communities. However, the parallel effect of diversity on resistance and recovery resulted in resilience (the ratio of recovery to resistance) to be independent of diversity. The lack of a diversity-resilience relationship, and the overall resilience of $\approx 1$, indicates that the productivity deviated from 

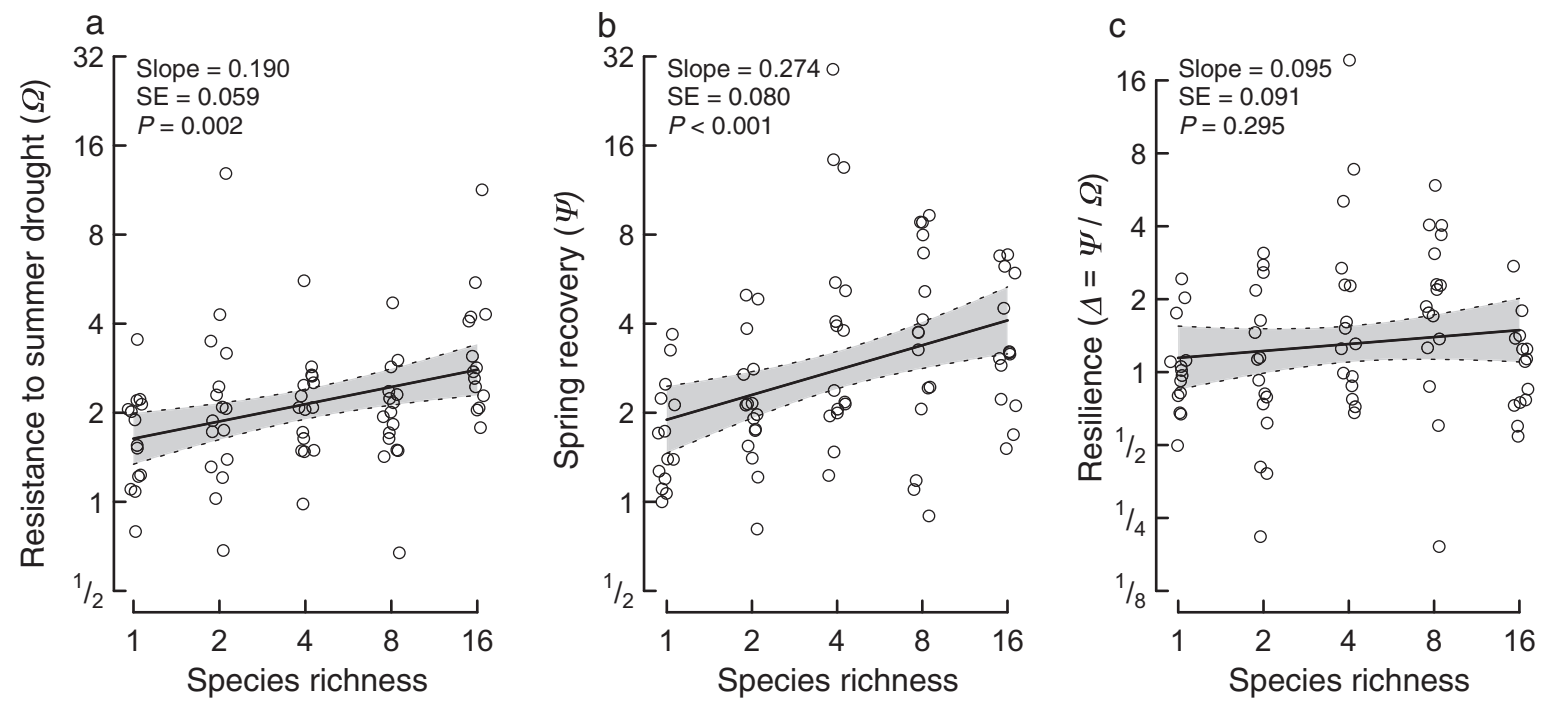

FIG. 5. Plant diversity effects on (a) resistance, (b) recovery and (c) resilience under recurrent summer droughts. Larger values indicate smaller deviations in productivity form ambient conditions. Slopes, standard errors (SE) and $P$-values for each relationship are provided. The solid line indicates the regression relationship and the grey-shaded region the $95 \%$ confidence interval. Species richness axes are log-scale.

ambient conditions relatively equally during summer droughts as it did in the spring following recovery, irrespective of diversity. This resulted because more diverse communities were productive beyond the productivity of communities experiencing the ambient state. Such increased productivity following a period of recovery has been reported previously (Vogel et al. 2012, Isbell et al. 2015, O'Brein et al. 2017). However, here we found that in more diverse communities the short-term increase in productivity following a period of recovery compensated for the lost productivity during summer on average. Consequently the compensatory recovery maintained the long-term productivity across years at higher diversity (Fig. 1d).

The increased variation in short-term seasonal productivity at higher diversity under drought compared to ambient conditions reflects short-term shifts in productivity in response to summer droughts. Our finding that increased spring productivity resulted from drought the previous summer is similar to observations that ecosystem primary productivity is related to the precipitation legacy experienced in previous years (Sala et al. 2012). However, no pattern in the compensatory recovery can be observed that corresponds to the precipitation patterns in our study and further data over more years are needed to explore the importance of precipitation-legacy effects.

The observed drought-induced, seasonal shifts in productivity are also likely related to drought effects on biogeochemical cycles and on the potential of plant communities to respond to the change in the availability of soil resources (Hofer et al. 2017, Mariotte et al. 2017). For instance, there are a growing number of studies that have shown that drought reduces the mobility of soil nutrients, litter decomposition, and the activity of soil microbes (Durand et al. 2010, Manzoni et al. 2012, Vogel et al. 2013, Hofer et al. 2017). Thus, under drought conditions, the plant-available soil resource pool becomes less accessible such that more resources are available post-drought to boost productivity once plant growth and soil biological activity has recommenced (Fierer and Schimel 2002, Hofer et al. 2017). Such carry-over legacies of drought on soil $\mathrm{N}$ availability have been observed to be the underlying mechanism behind the temporal changes in productivity in drylands and agriculture grassland ecosystems (Sala et al. 2012, Shen et al. 2016, Hofer et al. 2017).

Climatic perturbations such as droughts can shift the composition of the community structure and may alter the stabilizing role of species dominance and temporal asynchrony in performance among species (Schläpfer and Schmid 1999, Yachi and Loreau 1999, Loreau 2010, Yu et al. 2010). Although we could not assess this here, because no species-specific data are available for all years, we did observe that after seven years of recurring summer drought marginal species losses did occur. Thus, it is conceivable that drought-induced changes in the relative performance of species, and their interactions, that may have also played a role in the productivity of the communities. For instance, droughts are known to alter the species composition and the interactions among subordinate and dominant plant species (Kardol et al. 2010, Tucker et al. 2011, Mariotte et al. 2013, 2017). It has also been demonstrated that the compositional changes due to drought can favor more productive and stable dominant species that maintain the long-term productivity (Yu et al. 2010, 2015). Overall, the greater 
short-term seasonal variability, long-term stability, and greater resistance to drought at higher diversity in our study likely reflect that diversity provides ecosystems with an insurance to withstand climate change events.

In summary, we observed that drought increased the short-term variation in productivity in more diverse communities relative to ambient conditions. These short-term effects of recurrent droughts on seasonal productivity disappeared at the long-term scale. The shortterm destabilization in diverse communities resulted from compensatory recovery where more diverse communities increased productivity following a period of recovery to compensate for productivity losses during drought. As a result this compensatory recovery response stabilized the longer-term ecosystem functioning in more diverse communities. Our results provide a new example, and thus generalization for the "rule", that the stabilizing effect of diversity on ecosystem functioning at higher levels of organization, such as the community, occurs through increasing variation at lower levels of organization, such as species populations (Lehman and Tilman 2000, Flynn et al. 2008, Gonzalez and Loreau 2009, Loreau 2010, Wang and Loreau 2016). However, the buffering potential of biodiversity on ecosystem functioning under environmental perturbation may only work as long as the environmental perturbations do not lead to a reduction of biodiversity itself, which may happen under more severe droughts than we imposed. Whether biodiversity-driven buffering occurs in other systems over the long-term, or where thresholds are reached that lead to reductions in biodiversity itself, deserves further exploration. Overall, our results provide novel empirical insights into the importance of the timing and temporal scale at which the effects of environmental perturbations are observed to influence diversity-productivity or diversity-stability relationships (Peterson et al. 1998, Oliver et al. 2016).

\section{ACKNOWLEDGMENTS}

We thank Jørn Gandke, Victor Malakhov, Anne Ebeling and the Jena gardener team for assistance in maintaining the experiment and data collection as well as the reviewers and editor for the valuable time taken to provide insights to improve the manuscript. The Jena Experiment is funded by the German Research Foundation (FOR 456, FOR 1451). MOB was supported by the Stiefel-Zangger Fund through an SNF Mobility Grant (P2ZHP3_161986). NE acknowledges support by the German Centre for Integrative Biodiversity Research (iDiv) Halle-Jena-Leipzig, funded by the German Research Foundation (FZT 118). The authors declare no conflict of interest.

\section{Literature Cited}

Cardinale, B. J., et al. 2012. Biodiversity loss and its impact on humanity. Nature 486:59-67.

Dai, A. 2013. Increasing drought under global warming in observations and models. Nature Climate Change 3:52-58.

de Mazancourt, C., and M. Loreau. 2013. Biodiversity and ecosystem stability: a synthesis of underlying mechanisms. Ecology Letters 16:106-115.
Doak, D. F., D. Bigger, E. K. Harding, M. A. Marvier, R. E. O'Malley, and D. Thomson. 1998. The statistical inevitability of stability-diversity relationships in community ecology. American Naturalist 151:264-276.

Durand, J. L., V. Gonzalez-Dugo, and F. Gastal. 2010. How much do water deficits alter the nitrogen nutrition status of forage crops? Nutrient Cycling in Agroecosystems 88:231243.

Fierer, N., and J. P. Schimel. 2002. Effects of drying-rewetting frequency on soil carbon and nitrogen transformations. Soil Biology and Biochemistry 34:777-787.

Fischer, F. M., A. J. Wright, N. Eisenhauer, A. Ebeling, C. Roscher, H. de Kroon, C. Wagg, A. Weigelt, W. W. Weisser, and V. D. Pillar. 2016. Plant species richness and functional traits affect community stability after a flood event. Philosophical Transactions of the Royal Society B 317: 20150276.

Flynn, D. F. B., B. Schmid, J.-S. He, K. S. Wolfe-Bellin, and F. A. Bazzaz. 2008. Hierarchical reliability in experimental plant assemblages. Journal of Plant Ecology 1:59-65.

Folke, C., S. Carpenter, B. Walker, M. Scheffer, T. Elmqvist, L. Gunderson, and C. S. Holling. 2004. Regime shifts, resilience, and biodiversity in ecosystem management. Annual Review of Ecology, Evolution, and Systematics 35:557-581.

Gonzalez, A., and M. Loreau. 2009. The causes and consequences of compensatory dynamics in ecological communities. Annual Review of Ecology, Evolution, and Systematics 40:393-414.

Gross, K., J. B. Cardinale, J. W. Fox, A. Gonzalez, M. Loreau, H. W. Polley, P. B. Reich, and J. van Ruijven. 2014. Species richness and the temporal stability of biomass production: a new analysis of recent biodiversity experiments. American Naturalist 183:1-12.

Hodgson, D., J. L. McDonald, and D. J. Hosken. 2015. What do you mean, 'resilient'? Trends in Ecology and Evolution 30:503-506.

Hofer, D., M. Suter, N. Buchmann, and A. Lüscher. 2017. Nitrogen status of functionally different forage species explains resistance to severe drought and post-drought overcompensation. Agriculture, Ecosystem and Environment 236:312-322.

Hooper, D. U., E. C. A. Adair, B. J. Cardinale, J. E. K. Byrnes, B. A. Hungate, K. L. Matulich, A. Gonzalez, J. E. Duffy, L. Gamfeldt, and M. I. O'Connor. 2012. A global synthesis reveals biodiversity loss as a major driver of ecosystem change. Nature 486:105-108.

Isbell, F., et al. 2015. Biodiversity increases the resistance of ecosystem productivity to climate extremes. Nature 526: 524-577.

Kardol, P., C. E. Campany, L. Souza, R. J. Norby, J. F. Weltzin, and A. T. Classen. 2010. Climate change effects on plant biomass later dominance patterns and community evenness in an experimental old-field ecosystem. Global Change Biology 16:2676-2687.

Lehman, C. L., and D. Tilman. 2000. Biodiversity, stability, and productivity in competitive communities. American Naturalist 156:534-552.

Lehner, B., P. Döll, J. Alcamo, T. Henrichs, and F. Kaspar. 2006. Estimating the impact of global change on flood and drought risks in Europe: a continental integrated analysis. Climate Change 75:273-299.

Lloret, F., E. G. Keeling, and A. Sala. 2011. Components of tree resilience: effects of successive low-growth episodes in old ponderosa pine forests. Oikos 120:1909-1920.

Loreau, M. 2010. From populations to ecosystems: theoretical foundations for a new ecological synthesis (MPB-46). Princeton University Press, USA. 
Manzoni, S., J. P. Schimel, and A. Porporato. 2012. Responses of soil microbial communities to water stress: results from a meta-analysis. Ecology 93:930-938.

Mariotte, P., C. Vandenberghe, P. Kardol, F. Hagedorn, and A. Buttler. 2013. Subordinate plant species enhance community resistance against drought in semi-natural grasslands. Journal of Ecology 101:763-773.

Mariotte, P., A. Canarini, and F. A. Dijkstra. 2017. Stoichiometric N: P flexibility and mycorrhizal symbiosis favour plant resistance against drought. Journal of Ecology 105:958-967.

Mitchell, P. J., et al. 2016. An eco-climatic framework for evaluating the resilience of vegetation to water deficit. Global Change Biology 22:1677-1689.

Mori, A. S. 2016. Resilience in the studies of biodiversity-ecosystem functioning. Trends in Ecology and Evolution 31:87-89.

O'Brein, O. J., R. Ong, and G. Reynolds. 2017. Intra-annual plasticity of growth mediates drought resilience over multiple years in tropical seedling communities. Global Change Biology 23:4235-4244.

Oliver, T. H., et al. 2015. Biodiversity and the resilience of ecosystem services. Trends in Ecology and Evolution 30:673684.

Oliver, T. H., et al. 2016. A synthesis is emerging between biodiversity-ecosystem function and ecological resilience research: reply to Mori. Trends in Ecology and Evolution 31:189-192.

Peterson, G., C. R. Allen, and C. S. Holling. 1998. Ecological resilience, biodiversity, and scale. Ecosystems 1:6-18.

Pfisterer, A. B., and B. Schmid. 2002. Diversity-dependent production can decrease the stability of ecosystem functioning. Nature 416:84-86.

Pfisterer, A. B., J. Joshi, B. Schmid, and M. Fischer. 2004. Rapid decay of diversity-productivity relationships after invasion in experimental plant communities. Basic and Applied Ecology 5:5-14.

Roscher, C., J. Schumacher, J. Baade, W. Wilcke, G. Gleixner, W. W. Weisser, B. Schmid, and E.-D. Schulze. 2004. The role of biodiversity for element cycling and trophic interactions: an experimental approach in a grassland community. Basic and Applied Ecology 5:107-121.

Sala, O. E., L. A. Gherardi, L. Reichmann, E. Jobbagy, and D. Peters. 2012. Legacies of precipitation fluctuations on primary production: theory and data synthesis. Philosophical Transactions of the Royal Society B 367:3135-3144.

Schläpfer, F., and B. Schmid. 1999. Ecosystem effects of biodiversity: a classification of hypotheses and exploration of empirical results. Ecological Applications 9:893-912.

Schmid, B., M. Baruffol, Z. Wang, and P. A. Niklaus. 2017. A guide to analyzing biodiversity experiments. Journal of Plant Ecology 10:91-110.

Schröter, D., et al. 2005. Ecosystem service supply and vulnerability to global change in Europe. Science 310:1333-1337.
Shen, W., G. D. Jenerette, D. Hui, and R. L. Scott. 2016. Precipitation legacy effects on dryland ecosystem carbon fluxes: direction, magnitude and biogeochemical carryovers. Biogeosciences 13:425-439.

Skelton, R. P., A. G. West, and T. E. Dawson. 2015. Predicting plant vulnerability to drought in biodiverse regions using plant functional traits. Proceedings of the National Academy of Science of the United States of America 112:5744-5749.

Stocker, T. F., D. Qin, G.-K. Plattner, M. Tignor, S. K. Allen, J. Boschung, A. Nauels, Y. Xia, V. Bex, and P. M. Midgley. 2013. IPCC 2013: summary for policymakers. Cambridge University Press, UK.

Tilman, D. 1996. Biodiversity: population versus ecosystem stability. Ecology 77:350-363.

Tilman, D., and J. A. Downing. 1994. Biodiversity and stability in grasslands. Nature 367:363-365.

Tucker, S. S., J. M. Craine, and J. B. Nippert. 2011. Physiological drought tolerance and the structuring of tall grass prairie assemblages. Ecosphere 2:art48.

van Ruijven, J., and F. Berendse. 2010. Diversity enhances community recovery, but not resistance, after drought. Journal of Ecology 98:81-86.

Vogel, A., M. Scherer-Lorenzen, and A. Weigelt. 2012. Grassland resistance and resilience after drought depends on management intensity and species richness. PLoS ONE 7: e36992.

Vogel, A., N. Eisenhauer, A. Weigelt, and M. Scherer-Lorenzen. 2013. Plant diversity does not buffer drought effects on earlystage litter mass loss rates and microbial properties. Global Change Biology 19:2795-2803.

Wang, S., and M. Loreau. 2016. Biodiversity and ecosystem stability across scales in metacommunities. Ecology Letters 19:510-518.

Wang, Y., S. Yu, and J. Wang. 2007. Biomass-dependent susceptibility to drought in experimental grassland communities. Ecology Letters 10:401-410.

Weisser, W., et al. 2017. Biodiversity effects on ecosystem functioning in a 15-year grassland experiment: patterns, mechanisms, and open questions. Basic and Applied Ecology 23:1-17.

Yachi, S., and M. Loreau. 1999. Biodiversity and ecosystem productivity in a fluctuating environment: the insurance hypothesis. Proceedings of the National Academy of Science of the United States of America 96:1463-1468.

Yu, Q., Q. Chen, J. J. Elser, H. He, H. Wu, G. Zhang, J. Wu, Y. Bai, and X. Han. 2010. Linking stoichiometric homeostasis with ecosystem functioning and stability. Ecology Letters 13: 1390-1399.

Yu, Q., K. Wilcox, K. La Pierre, A. K. Knapp, X. Han, and M. D. Smith. 2015. Stoichiometric homeostasis predicts plant species dominance, temporal stability, and responses to global change. Ecology 96:2328-2335.

\section{SUPPORTING INFORMATION}

Additional supporting information may be found in the online version of this article at http://onlinelibrary.wiley.com/doi/ 10.1002/ecy.2003/suppinfo 